\title{
Diagnostic Art: A Tribute to Professor Gérard Simon
}

\section{J. Allan Tucker}

\section{Department of Pathology, University of South Alabama, Mobile, Alabama, USA}

Dr. Gérard Simon enjoyed a highly successful and memorable career in electron microscopy, and was a founding member and major contributor to the Microscopical Society of Canada, which currently offers two awards in his honor. In his leisure time, he enjoyed painting and sketching. This presentation in his honor explores an experience in both fine art and in the art of electron microscopy.

In diagnostic pathology, ultrastructural findings are utilized to gain an impression of a disease state. As one example, a two year-old boy presented with a perplexing mass in the right mastoid region. A small biopsy was performed, and the light microscopy revealed a small blue cell tumor. Lymphoma was suspected, and a number of immunohistochemical studies were performed. These were not diagnostic, and regrettably depleted the block. Fortunately, a tiny aliquot of the biopsy had been submitted for electron microscopy. Ultrastructural examination revealed tumor cells with cell processes containing numerous microtubules. This appearance alone would be diagnostic of neuroblastoma, but in addition, numerous neurosecretory granules could be identified. As would not have otherwise been possible, a definitive diagnosis of neuroblastoma was rendered, which was clinically unsuspected.

Impressions also exist as works of art - prints produced by pressing paper against an inked matrix. This technique provided artists with the opportunity to produce many art products from a single effort prior to the development of other techniques to reproduce works. One of the best-known artists to utilize impressions specifically etchings - was Rembrandt. He had a room in his home with a press to produce etchings. His last documented etching, produced in about 1665, was a portrait of the physician Jan Antonides van der Linden, and was intended as the frontispiece for a book on Hippocrates by that physician, but was not used.

Continuing on the theme of impressions, one major post-impressionistic artist was Henri de ToulouseLautrec. This artist suffered from pyknodysostosis, resulting in a short stature. As a result, he often did not include the full length of subjects' legs in his works. While known for his excesses - he died of alcoholism and syphilis - he had an interest in medicine. His works include a drawing of the surgeon Jules-Émile Péan, inventor of the hemostat.

As another example of an ultrastructural impression, a 79 year-old man presented with a $4 \mathrm{~cm}$ testis mass. He underwent unilateral orchiectomy, and the testis contained an epithelioid tumor suspected to represent some sort of germ cell tumor, but the light microscopy and immunohistochemistry were inconclusive. The case was sent in consultation. While no tissue had been submitted in glutaraldehyde, electron microscopy on formalin-retrieved tissue revealed that the tumor cells exhibited long, sometimes largely circumferential microvilli, and tonofilament bundles were seen. The possibility of a tumor growing into the testis had not been considered, but these finding revealed that the lesion actually represented a malignant mesothelioma of the tunica albuginea.

Regarding impressions, one particularly noteworthy art period was that of the Impressionists. These artists painted more of an impression of the subject rather than an attempt to replicate it. Interestingly, the term originated from a derisive review of their work. One of these artists was Berthe Morisot. These artists knew one another well; Morisot married Eugène Manet, brother of the artist Édouard Manet, so her daughter Julie was Julie Manet (Morisot's only child). She sometimes used her daughter and a niece as her subjects. Further, she was good friends with Pierre-Auguste Renoir, and they sometimes traveled 
together. He completed a number of works featuring these same two girls. Morisot and Renoir were such good friends that when Morisot died at a young age, having already lost her husband, she named Renoir as her daughter's guardian.

Other areas of tumor diagnosis in which the ultrastructural impression can be critical include alveolar soft part sarcoma and acinar cell carcinoma. Another artist along the previous theme is Jean-Baptiste-Camille Corot, a teacher of Morisot. And Pablo Picasso, an artist and philosopher, left a lasting impression.
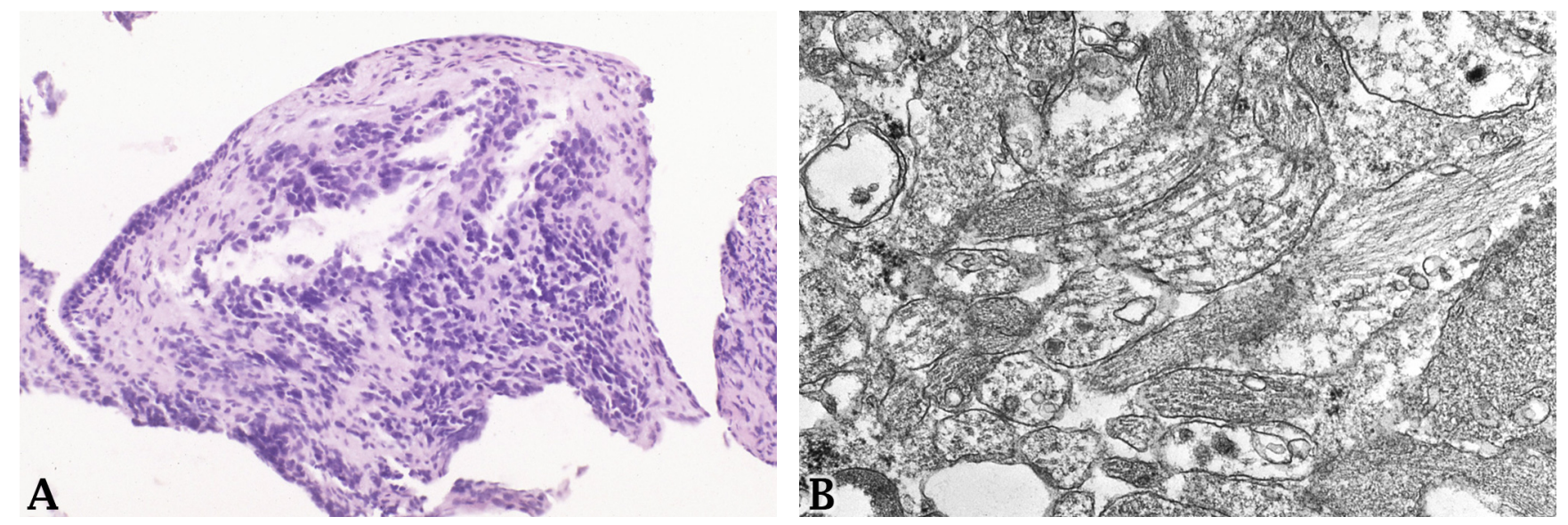

Figure 1. A. Small biopsy of a mastoid lesion in a two year-old boy. The light microscopy and immunostains were inconclusive. B. Electron microscopy revealed cell processes with microtubules; the impression gained from electron microscopy was diagnostic of neuroblastoma.

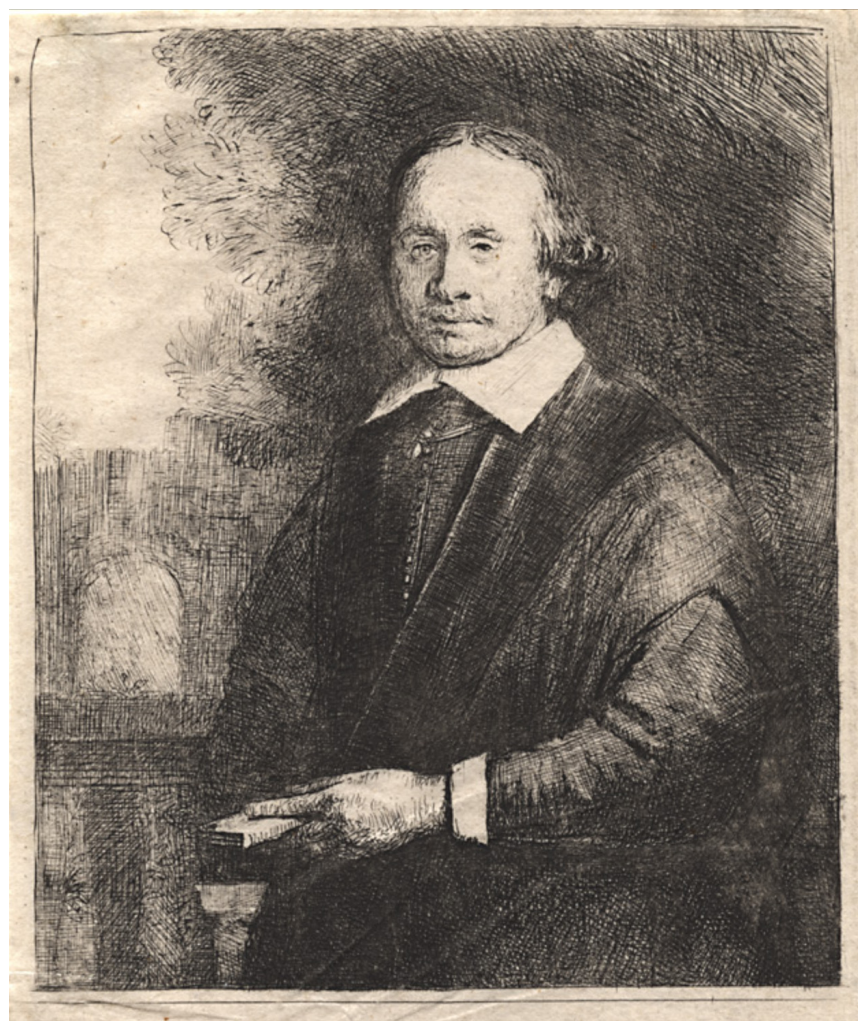

Figure 2. An impression (etching) by Rembrandt of the physician Jan Antonides van der Linden. 